\title{
CHK2 sets the stage for CK1 in oocyte quality control
}

\author{
Sebastian Kehrloesser ${ }^{1,2} \cdot$ Marcel Tuppi $\mathbb{I I}^{1} \cdot$ Volker Dötsch $(\mathbb{1})^{1}$
}

Published online: 17 April 2018

(c) ADMC Associazione Differenziamento e Morte Cellulare 2018

TAp63 $\alpha$ has been identified as the major quality control factor in the female germline more than a decade ago by the group of Frank McKeon. DNA damage in primary oocytes, within primordial follicles, that constitute the ovarian reserve in women, results in the phosphorylation-dependent activation of p63 that in turn induces apoptosis of the affected oocytes [1]. Although this mechanism is crucial to ensure oocyte quality throughout the reproductive lifespan, female cancer patients treated with DNA damaging chemotherapy or pelvic radiotherapy frequently suffer from premature ovarian insufficiency (POI) and its detrimental side effects due to severe reduction of the primordial follicle pool. To develop strategies to protect primary oocytes during gonadotoxic therapies and thus prevent POI, it is important to understand the molecular details of TAp $63 \alpha$ regulation. Women are born with a finite number of oocytes that are arrested in prophase of meiosis I until recruited for ovulation-a period that can last for decades $[2,3]$. The constitutively high expression of an apoptosis-inducing transcription factor combined with this long arrest time necessitates a very tight regulation of the transcriptional activity of TAp63 [4]. Our group has previously demonstrated that in contrast to all other p53 family members [5], TAp63 $\alpha$ adopts an autoinhibited and only dimeric conformation [6]. DNA damage-induced phosphorylation serves as a trigger that disrupts this kinetically trapped conformation allowing transition from the inactive dimer to

These authors contributed equally: Kehrloesser Sebastian,Tuppi Marcel.

Volker Dötsch

vdoetsch@em.uni-frankfurt.de

1 Institute of Biophysical Chemistry and Center for Biomolecular Magnetic Resonance, Goethe University, Frankfurt/ Main, Germany

2 Present address: Li Ka Shing Centre, CRUK Cambridge Institute, University of Cambridge, Robinson Way, Cambridge, CB2 ORE, UK the active tetramer with a 20 -fold higher DNA binding affinity [7]. Activated TAp63 $\alpha$ then orchestrates the induction of apoptosis by driving transcription of genes coding for the $\mathrm{BH} 3$ only proteins PUMA and NOXA [8]. Although several kinases and phosphorylation sites have been suggested, the exact activation mechanism for TAp63 $\alpha$ remained enigmatic and in part controversially discussed $[9,10]$. Although CHK2 has been identified as a kinase essential for activation of TAp63 $\alpha$ [11], we report now that phosphorylation at S582 is essential but not sufficient to transform the protein into the active tetramer [7]. Activation of TAp63 $\alpha$ requires a two-step mechanism that involves priming by $\mathrm{CHK} 2$ and subsequent secondary phosphorylation events by the executioner kinase CK1 (S585-T594) [12]. This accumulation of phosphate groups in the C-terminal region leads to electrostatic repulsion of a spatially close acidic sequence disrupting the autoinhibitory structure (Fig. 1). Our results thus provide a structural rationale for the phosphorylation-induced activation of p63 and further demonstrate that the previously suggested and controversially discussed tyrosine phosphorylation by c-Abl $[9,10]$ is in fact dispensable for the activation mechanism. The involvement of two different kinases also explains how oocytes achieve the tight regulation of p63 tetramerization that suppresses accidental activation and subsequent oocyte death. The requirement for multiple phosphorylation events by two different kinases ensures that only a strong activation of the DNA damage response leads to activation of TAp63 $\alpha$ while sporadic phosphorylation could be efficiently removed by phosphatases [13]. In agreement with our model, death of primary oocytes in cultured mouse ovaries treated with the common chemotherapeutic agents doxorubicin or cisplatin could be prevented by coadministration of kinase inhibitors targeting either CHK2 or $\mathrm{CK} 1$, but not with the c-Abl inhibitor imatinib. Identification of CK1 as a second essential kinase adds a valuable target for the development of fertoprotective strategies. As the fertoprotective therapy must not interfere with the actual tumor- targeting therapy regime or even promote tumor progression, it is unlikely that a single fertoprotective 


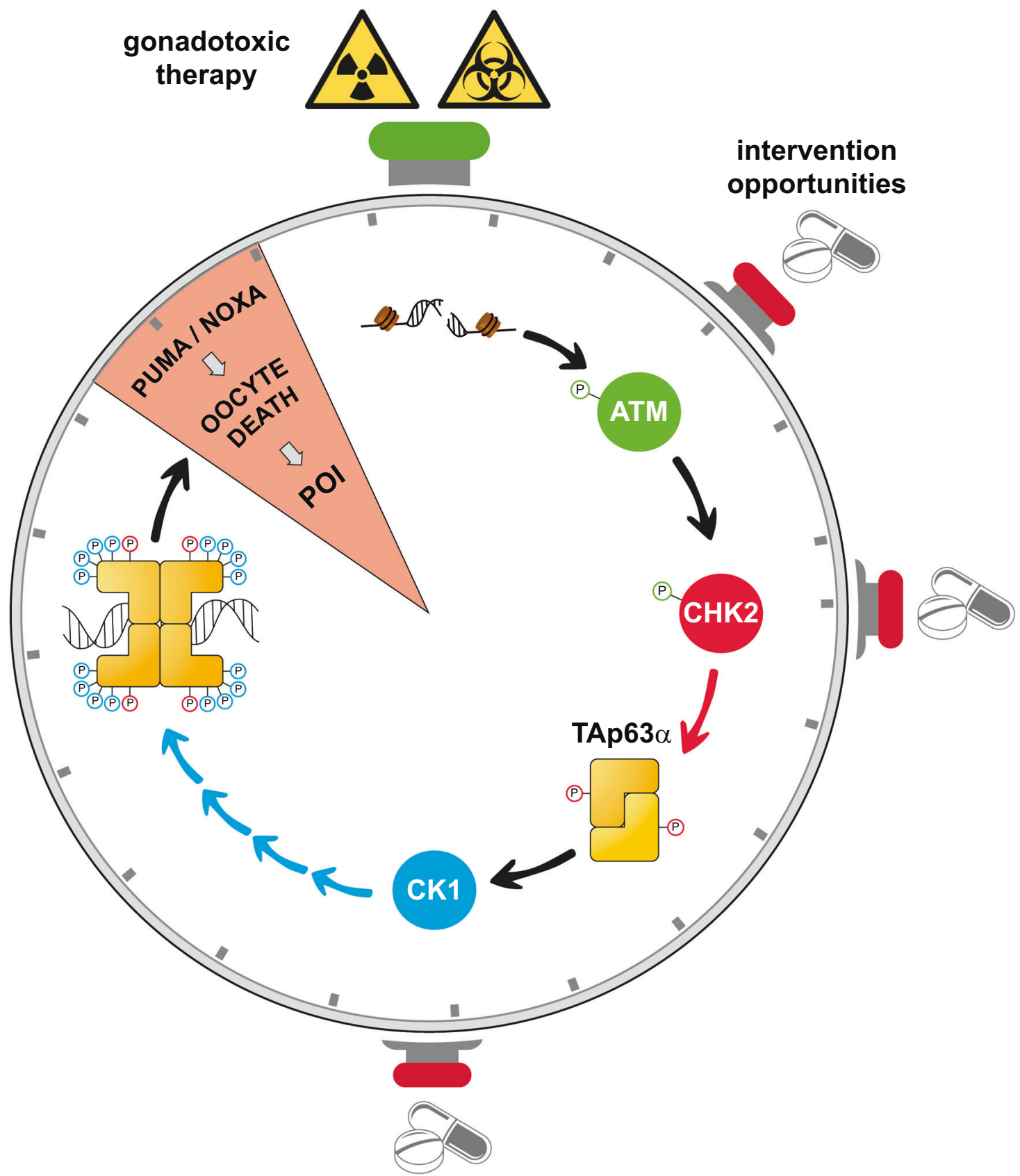

Fig. 1 Activation of TAp63 $\alpha$ in primary oocytes requires a two-step mechanism, which works as a molecular fuse

strategy will be suitable for all cancer types. Therefore, it will be important to identify multiple targets for tailoring fertoprotective therapies for the exact cancer type and treatment regime. In addition to the kinase inhibitors mentioned above several hormones and small molecules have been proposed as fertoprotective agents (e.g., luteinizing hormone, sphingosine-1-phosphate, AS101) [14, 15]. Their mechanism of action, however, remains elusive at the moment and it will be crucial to understand whether, how and at what stage these compounds interfere with p63 activation or its downstream signaling.
Chemotherapy-induced DNA damage activates ATM, which subsequently activates CHK2. Active CHK2 acts as an essential priming kinase and phosphorylates TAp63 $\alpha$, which is highly expressed in its autoinhibited dimeric conformation, on S582. This sets the stage for the executioner kinase CK1, which requires a pre-phosphorylated substrate, to add up to four additional phosphate groups C-terminally (S585, S588, S591, and T594) to the CHK2 site. The sum of these phosphorylation events disrupts the autoinhibited dimeric conformation and allows TAp63 $\alpha$ to form an open and active tetramer. Tetrameric 
TAp63 $\alpha$ is able to induce apoptosis by driving expression of PUMA and NOXA, which will eventually result in POI of the patient.

Acknowledgements The research was funded by the DFG (DO 545/81), the Centre for Biomolecular Magnetic Resonance (BMRZ), and the Cluster of Excellence Frankfurt (Macromolecular Complexes). MT was supported by a Fellowship from the Fonds of the Chemical Industry and Boehringer Ingelheim Fond travel grant.

\section{Compliance with ethical standards}

Conflict of interest The authors declare that they have no conflict of interest.

\section{References}

1. Suh E-K, et al. p63 protects the female germ line during meiotic arrest. Nature. 2006;444:624-8.

2. Johnston RJ, Wallace WHB. Normal ovarian function and assessment of ovarian reserve in the survivor of childhood cancer. in Pediatr Blood Cancer. 2009;53:296-302.

3. Gebel J, et al. Control mechanisms in germ cells mediated by p53 family proteins. J Cell Sci. 2017;130:2663-71.

4. Amelio I, Grespi F, Annicchiarico-Petruzzelli M, Melino G. p63 the guardian of human reproduction. Cell Cycle. 2012;11:4545-51.

5. Luh LM, et al. Analysis of the oligomeric state and transactivation potential of TAp73 $\alpha$. Cell Death Differ. 2013;20:1008-16.
6. Deutsch GB, et al. DNA damage in oocytes induces a switch of the quality control factor TAp63 $\alpha$ from dimer to tetramer. Cell. 2011;144:566-76.

7. Coutandin D, et al. Quality control in oocytes by p63 is based on a spring-loaded activation mechanism on the molecular and cellular level. eLife. 2016;5:e13909.

8. Kerr JB, et al. DNA damage-induced primordial follicle oocyte apoptosis and loss of fertility require TAp63-Mediated induction of puma and noxa. Mol Cell. 2012;48:343-52.

9. Gonfloni S, et al. Inhibition of the c-Abl-TAp63 pathway protects mouse oocytes from chemotherapy-induced death. Nat Med. 2009; 15:1179-85.

10. Kerr JB, et al. Cisplatin-induced primordial follicle oocyte killing and loss of fertility are not prevented by imatinib. Nat Med. 2012;18:1170-2-4.

11. Ewelina B-F, Vera DR, Michelle EW, Schimenti JC. Reversal of female infertility by Chk2 ablation reveals the oocyte DNA damage checkpoint pathway. Science. 2014;343:533-6.

12. Tuppi $\mathrm{M}$, et al. Oocyte DNA damage quality control requires consecutive interplay of CHK2 and CK1 to activate p63. Nat. Struct. Mol. Biol. 2018; https://doi.org/10.1038/s41594-0180035-7.

13. Kim D-A, Suh E-K. Defying DNA double-strand break-induced death during prophase I meiosis by temporal TAp63 $\alpha$ phosphorylation regulation in developing mouse oocytes. Mol Cell Biol. 2014;34:1460-73

14. De Vos M, Smitz J, Woodruff TK. Fertility preservation in women with cancer. Lancet. 2014;384:1302-10.

15. Rossi V, et al. LH prevents cisplatin-induced apoptosis in oocytes and preserves female fertility in mouse. Cell Death Differ. 2017;24:72-82. 